Editorial

\title{
Are Probiotics Pro-Obesity or Potential Anti-Obesity Agents?
}

\section{Farid Menaa}

Department of Health and Biosystems, Fluorotronics Inc and California Innovation Corp, San Diego, California, USA.

Corresponding author: Farid Menaa, Director \& Consulting Expert, Department of Health and Biosystems, Fluorotronics Inc and California Innovation Corp, San Diego, California, USA. E-mail: dr.fmenaa@gmail.com

Received Date: March 12, 2015

Accepted Date: March 12, 2015

Published Date: March 14, 2015

Citation: Menaa, F. Are Probiotics Pro-Obesity or Potential Anti-Obesity Agents? (2015) J Food Nutr Sci 2(1): 1.

\section{Editorial}

Since the alarming report made by the French Scientist Professor Dr Didier Raoult in $2009^{[1]}$, this topic remains a debate, especially between the scientific community and the agroindustry.

Are probiotics, before used as growth factors for livestock $^{[2]}$ healthy or do they contribute to inflammatory-state diseases such as obesity? What is the exact composition of microbiota, in terms of quantity and quality that could be considered as healthy? How probiotics regulates the host-microbiotal crosstalk (bacteriocin production)? Shall we, as consumers, believe that some products such as "Activia" or Actimel" are deleterious for our health or shall be recommended to anybody? Shall the agroindustry be pressed to provide transparent information (e.g. quantity of probiotics in a milk-based product) for the consumer instead of beating around the bush? It seems that a wreck falls into our yoghurt pot!

Well, It is increasingly becoming clearer that gut microbiota represents a key player both in health and disease depending on its level of (good or bad) alterations ${ }^{[3,4]}$ which subsequently affect the body metabolism. Albeit the molecular mechanisms have not been fully established, alterations in gut microbiota composition by means of probiotics, prebiotics or antibiotics have been reported to be associated with plenty disorders such as obesity ${ }^{[3]}$. Obesity, a serious public health problem, defined as abnormal or excessive fat accumulation that presents a risk to health, that has reached epidemic proportions worldwide, particularly in urban settings and children populations ${ }^{[5]}$. A crude population measure of obesity is the body mass index (BMI), a person's weight (in kilograms) divided by the square of his or her height (in meters) ${ }^{[5]}$. Thereby, a person with a BMI of 30 or more is generally considered obese while a person with a BMI equal to or more than 25 is considered overweight ${ }^{[5]}$. One major fact is that management of obesity is quite expensive, notably due to its comorbidities such as diabetes (e.g. T2D), fatty liver, metabolic syndrome, cardiovascular diseases (CVDs) and cancers $^{[5,3]}$. In human beings, as in animals, specific probiotics are associated with weight gain or loss ${ }^{[4]}$. The determination of exact obesity-related mechanisms underlying cross-talks between gut microbiota and probiotics as well as the right dose and composition of probiotics would be an asset in personalized medicine, especially to treat childhood obesity worldwide.

Acknowledgment: The author thanks Dr. Abder Menaa, MD, specialist in Nutrition, for our fruitful discussions on this important public health matter.

\section{References}

1. Raoult, D. Probiotics and obesity: a link? (2009) Nat Rev Microbiol 7(9): 616.

2. Khan, M., Raoult, D., Richet, H., et al. Growth-promoting effects of single-dose intragastrically administered probiotics in chickens. (2007) Br Poult Sci 48(6): 732-735.

3. Villanueva-Millán, M. J., Pérez-Matute, P., Oteo, J. A. Gut microbiota: a key player in health and disease. A review focused on obesity. (2015) J Physiol Biochem.

4. Angelakis, E., Merhej, V., Raoult, D. Related actions of probiotics and antibiotics on gut microbiota and weight modification. (2013) Lancet Infect Dis 13(10): 889-899.

5. World Health Organization (2015).

Copy rights: (C2015 Menaa, F. This is an Open access article distributed under the terms of Creative Commons Attribution 4.0 International License. 